\title{
Lateral Symmetry of Center of Pressure During Walking in Patients With Unilateral Knee Osteoarthritis
}

\author{
Si-hyun Kim¹, PT, PhD, Kyue-nam Park², PT, PhD \\ ${ }^{1}$ Department of Physical Therapy, Sangji University, Wonju, ${ }^{2}$ Department of Physical Therapy, College of Medical Science, Jeonju University, \\ Jeonju, Korea
}

\author{
Article Info \\ Received January 18, 2021 \\ Revised January 23, 2021 \\ Accepted January 26, 2021 \\ Corresponding Author \\ Kyue-nam Park \\ E-mail: knpark@jj.ac.kr \\ https://orcid.org/0000-0003-3521-3121
}

\section{Key Words \\ Gait analysis \\ Knee \\ Osteoarthritis \\ Walking}

Background: Although symmetry of spatio-temporal parameter and center of pressure (COP) shift during walking is associated with knee adduction moment, research on clinical association with knee osteoarthritis (OA)-related knee pain and functional scores is lacking.

Objects: The aims were 1) to compare symmetry of gait parameters and COP-shift in patients with unilateral knee OA and pain and matched controls, and 2) to investigate the relationship between symmetry of gait parameters and COP-shift, and clinical measures.

Methods: Female subjects ( $n=16$ ) had with unilateral radiological knee OA and pain. Healthy controls ( $n=15$ ) were age-matched to OA group. Symmetry of foot rotation, step length, stance and swing phase, lateral symmetry of COP and anterior/posterior symmetry of COP during walking was assessed. To assess the clinical variables, pain intensity, pain duration and function using Knee Osteoarthritis Outcome Survey (KOOS) subscales were collected. We compared symmetry between groups using Mann-Whitney U-test or independent t-test. Relationships between clinical measures and symmetry index measured using Spearman's correlation test. Statistical significance was set at $\alpha=0.05$.

Results: Knee OA group showed significantly greater values of only lateral symmetry of COP $(p<0.01)$ than healthy group. Values of lateral symmetry of COP had moderate or strong correlation significantly with the intensity of knee pain, pain duration, and scores of all KOOS subscales $(p<0.01)$.

Conclusion: Patients with unilateral knee OA and pain showed more asymmetry of lateral COP-shift during walking compared with matched healthy controls. In addition, larger asymmetry of lateral COP-shift has the moderate or strong association with worse of knee pain, worse in KOOS scores and longer duration of knee pain. Asymmetry of lateral COP-shift during walking may be one of the characteristics of unilateral knee $\mathrm{OA}$ as the compensatory strategy response to unilateral OA of the knee.

\section{INTRODUCTION}

Knee osteoarthritis (OA) is one of the source of the disability in older population, and prevalence of knee OA is significantly higher and radiographic sign of knee OA is more severe in females than males [1,2]. Patients with more severe knee OA showed more alteration in frontal kinematic of knee, such as knee adduction during gait than healthy older and patients with less severe knee OA [3]. To attenuate external knee adduction moment and medial knee stress of more symptomatic side during walking, patients with knee OA increase toe-out angle and lateral shift of the trunk and shift the body weight side- to-side more rapidly to alter medial tibiofemoral stress than healthy controls [3]. These gait alterations can place the greater stress on the knee joint of the less symptomatic or non-painful side due to asymmetrical load distribution, that is related with increasing the risk of OA development in less symptomatic or non-painful side in patients with unilateral knee OA [4].

Some study reported that OA tend to occur asymmetrically, while rheumatoid arthritis tends to occur symmetrically [5]. Symmetry or asymmetry of gait parameters has been used to distinguish between healthy and patients and what stage a patient is in specific disease including knee OA [6,7]. Gait asymmetry was defined as the different patterns of the kinetic, 
kinematic and spatio-temporal parameters, as a result of gait adaptation response to knee OA [8,9]. Patients with either unilateral or bilateral knee OA have asymmetrical angle of knee varus during walking [9]. However, when dividing the patients with knee OA to two groups according to unilateral or bilateral knee pain, OA patients with bilateral pain showed symmetrical angle of knee varus, while OA patients with unilateral pain showed asymmetrical angle of knee varus [10].

Shifts of the center of pressure (COP) affect the ground reaction force, leading to impact loading to the knee joint [11]. However, there is controversy over whether symmetry of COP is differed between older people with and without knee OA. A previous study demonstrated that laterality of COP of patients with knee OA is greater than healthy control [12]. Less lateralization of COP, less the knee adduction moment, while more COP-shift laterally, more the knee adduction moment increases [11]. In the other hands, another previous study reported that the amounts of anterior/posterior COP-shift was less in patients with knee OA than control group when standing both affected and sound leg, while no differences in lateral COPshift between groups in initial phase of walking [13].

If symmetry of gait parameters and COP-shift will be related with pain, symptom and function of knee OA in patients with both unilateral knee OA and unilateral pain, the current study sheds some light on the characteristics relating symmetry of COP-shift during walking in patients with symptomatic knee OA for physiotherapist who assess the quality of gait and train the symmetrical gait pattern to patients. The aims of current study are 1) to compare the symmetry of gait parameters and COP-shift antero-posteriorly and laterally in female older patients with unilateral knee OA and pain and matched controls, and 2) to investigate the relationship between symmetry of gait parameters and COP-shift and clinical measures such as pain intensity, pain duration, and functional scores related knee OA. Our hypotheses are that knee OA group showed more asymmetry than controls and symmetry of COP-shift will be associated with clinical measures.

\section{MATERIALS AND METHODS}

\section{Subjects}

Fifty-one female subjects with and without knee pain during walking volunteered from the welfare center in Jeonju. Twenty of the 51 female volunteers did not meet the inclusion and exclusion criteria due to following reasons: Kellgren-Lawrence grade of $\mathrm{OA}<2$ in 5 volunteers, bilateral knee $\mathrm{OA}$ in 11 volunteers and unilateral left knee OA in 4 volunteers. The thirtyone subjects (sixteen for knee OA group and fifteen for healthy control group) met the criteria (Figure 1). The sample size of at least 15 subjects produced statistical power of 0.80 , assuming medium effect size $(d=0.6)$ and a level of significance of $5 \%$ [14]. Inclusion criteria for knee OA group $(n=16)$ were that 1$)$ age was over fifty years old, 2) recent weight-bearing posterioanterior radiographic confirmation of unilateral right knee OA clinical diagnosis by an OA rheumatologist, 3) a Kellgren-Lawrence grade of $\mathrm{OA}$ was over 2 and 4) unilateral right knee pain was over 3 on a $10 \mathrm{~cm}$ visual analogue scale (VAS) on most days of the previous week during walking [6,10]. Subjects in knee OA group were excluded from current study if they 1) had left knee OA or bilateral knee OA, 2) had taken oral corticosteroids in the past 4 weeks, 3) corticosteroid injections within the past 3 months prior to testing, 4) had undergone total knee

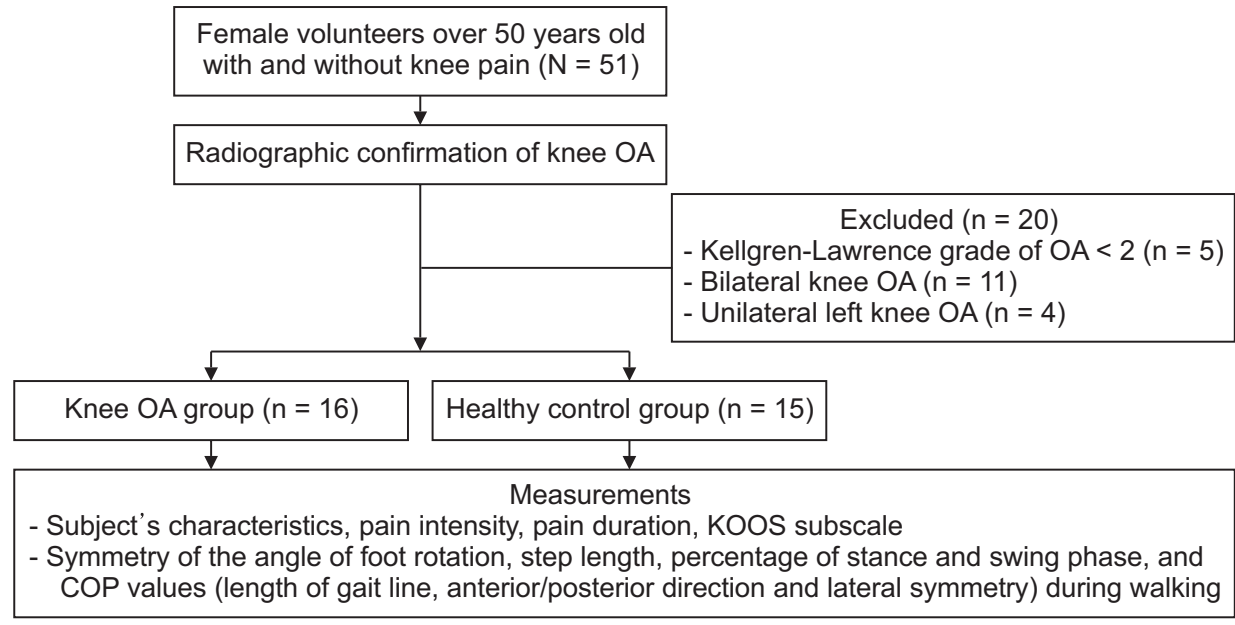

Figure 1. Flow chart of the study. OA, osteoarthritis; KOOS, Knee Osteoarthritis Outcome Survey; COP, center of pressure. 
arthroplasty, 5) had OA in lumbar and lower limb joints except of knee, 6) had a body mass index of $>33 \mathrm{~kg} / \mathrm{m}^{2}$, 6) had systemic arthritic conditions, and cognitive, cardiopulmonary and neurological impairments that would prevent participation, and 7) were not able to walk without assistive device [6,15].

Fifteen female subjects participated in age-matched control group. Inclusion criteria for the control group 1) were no signs of knee OA based on radiographic confirmation of knee OA by an $\mathrm{OA}$ rheumatologist and 2) were no pain of the knee during gait. This study was approved by the Jeonju University Institutional Review Board (jjIRB-161108-HR-2016-1102). Prior to current study, all experimental procedures were explained to each subject in detail. All subjects provided written informed consent.

\section{Instrumentation}

\section{1) Knee Osteoarthritis Outcome Survey}

The Knee Osteoarthritis Outcome Survey (KOOS) is selfreported questionnaire with high validity and reliability (intraclass correlation coefficients $=0.78-0.97$ ) in patients with $\mathrm{OA}$ in order to assess the opinion of OA patients about knee and associated problems [16]. KOOS holds 42 items with five subscales (pain, symptoms, activities of daily living, function in sport and recreation, and knee related quality of life). A Likert scale is used and all items scored from 0 (No Problems) to 4 (Extreme Problems). Scores are transformed to a 0-100 scale using Microsoft Excel, downloaded from http://www.koos.nu and ranged from 0 to 100 with higher scores indicating fewer knee problems [16].

\section{2) Center of pressure measurement system}

Zebris-FDM 1.5 force distribution measuring plate (ZEBRIS $\mathrm{GmbH}$, Isny, Germany) with 11,264 force sensors was used to measure the angle of foot rotation, step length, rate of stance, and swing phase and COP during walking. This system have high levels of within-day reliability for measuring gait parameters with coefficients of variation typically below 7\% [17]. Two units of the Zebris-FDM 1.5 were connected and made a 3-meter long measuring plate. Measuring plates were extended with an additional 1.5-meter track for the start and end points. A sampling rate of $100 \mathrm{~Hz}$. High density of pressure sensors can map to visualize subtle changes in force distribution during gait. WinPDMS ver. 1.2.2 measuring software provides two or three dimensional graphic pattern of COP trajectories and generate a "butterfly" pattern, which show a continuous COP trace during walking. Three parameters were derived from the butterfly; (1) length of gait line ( $\mathrm{mm})$ : the progression of the COP of all steps recorded of one side of the body, (2) anterior/ posterior direction (mm): defined as the shift of the COP intersection point towards forwards or backwards direction, "zero position" means the rearmost place where the heel contacts the ground, and (3) lateral symmetry ( $\mathrm{mm})$ : the shift of the COP intersection point towards left/right direction; "zero position" indicate perfect symmetry (Figure 2). Negative value means a shift to the left, and a positive value means a shift to the right.

\section{Procedure}

Following anthropometric assessment, all subjects reported their average knee pain during walking over the past week on a VAS and completed the KOOS. Subjects in healthy group also completed the KOOS, because none of the KOOS scores reached the maximum value (100) [18]. For familiarization, all subjects walked barefoot at individualization, all subjects walked over the FDM-S pressure mat. And then, ten gait trials were performed for each subject and recorded the data in the middle of eight gait trial to consider the effects of initiation and termination of the gait $[19,20]$. Symmetry of COP values (anterior/posterior direction and lateral symmetry) was showed automatically in proprietary software. The software was also used to show the angle of foot rotation, step length, percentage of stance, and swing phase. In order to calculate the symmetry of the angle of foot rotation, step length, percentage of stance and swing phase, and length of gait line between both side of the leg, current study used symmetry index, that is used as the most sensitive measurement of symmetry of the gait in regarding to spatial-temporal parameters [20]. The symmetry index

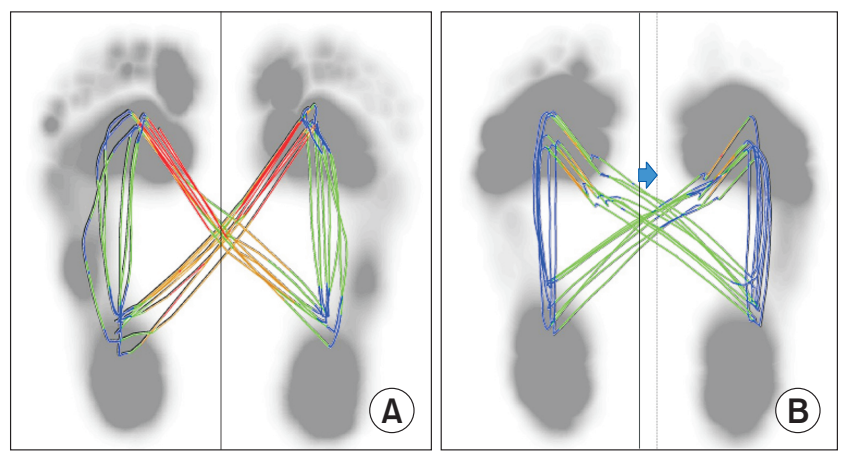

Figure 2. Butterfly diagrams of lateral symmetry of center of pressure during walking: (A) Healthy subject, (B) Patient with unilateral knee osteoarthritis. 
was a percentage assessment, which was calculated by the differences of gait parameters between both lower limbs against average value. The value of symmetry index $=0$ indicates full symmetry. The formula of symmetry index $=\left|\left(X_{\text {side1 }}-X_{\text {side } 2}\right)\right| /$ $\left[0.5 \times\left(X_{\text {sidel } 1}+X_{\text {sidee }}\right)\right] \times 100[21]$.

\section{Statistical analysis}

As a result of Kolmogorov-Smirnov tests for evaluation of the normality of each variable, all symmetry index of gait parameters and COP were non-parametric data except of the data of anterior/posterior position of COP. Anthropometric data and scores of each subscale of KOOS between groups were evaluated using Mann-Whitney U-test. We compared the betweenlimb symmetry of angle of foot rotation, step length, swing phase, stance phase, length of gait line, lateral symmetry of COP and anterior/posterior position of COP between patients with knee OA and healthy controls using Mann-Whitney U-test or independent t-test. Relationships between clinical measures and values of symmetry index measured using Spearman's correlation test. Level of Spearman's correlation was ranged as follows; weak level 0.20-0.39, moderate level 0.40-0.59, strong level $0.60-0.79$ and very strong $0.80-1.0$. The level of significance was set at $\alpha=0.05$. The SPSS 21 (IBM Co., Armonk, NY, USA) was used for statistical analysis.

\section{RESULTS}

Characteristics of the subjects, stratified by group, are shown

Table 1. Subject's characteristics and clinical measures

\begin{tabular}{lccc}
\hline \multicolumn{1}{c}{ Characteristics } & $\begin{array}{c}\text { Knee OA } \\
\text { group }\end{array}$ & $\begin{array}{c}\text { Healthy control } \\
\text { group }\end{array}$ & p-value \\
\hline Number of subjects (female) & 16 & 15 & - \\
Painful side & $\begin{array}{c}\text { Right }(\mathrm{n}=16) \\
\text { Age }(\mathrm{y})\end{array}$ & - & - \\
Height $(\mathrm{m})$ & $156.9 \pm 7.4$ & $60.0 \pm 3.3$ & 0.40 \\
Weight $(\mathrm{kg})$ & $60.5 \pm 11.5$ & $157.6 \pm 5.4$ & 0.59 \\
Body mass index $\left(\mathrm{kg} / \mathrm{m}^{2}\right.$ ) & $24.7 \pm 4.5$ & $23.1 \pm 2.0$ & 0.26 \\
Waist circumference $(\mathrm{cm})$ & $90.6 \pm 9.0$ & $86.9 \pm 5.4$ & 0.20 \\
Level of knee OA $_{\text {Intensity of knee pain }(\mathrm{cm})}$ & $2.37 \pm 0.9$ & $1.3 \pm 0.7$ & $<0.01$ \\
Pain duration (months) & $6.1 \pm 1.8$ & $0.0 \pm 0.0$ & $<0.01$ \\
Symptoms & $52.2 \pm 41.0$ & $0.0 \pm 0.0$ & $<0.01$ \\
Pain $^{\mathrm{a}}$ & $42.6 \pm 12.3$ & $94.5 \pm 2.7$ & $<0.01$ \\
Activities of daily living $^{\mathrm{a}}$ & $42.0 \pm 14.8$ & $97.0 \pm 1.9$ & $<0.01$ \\
Sport and recreation $^{\mathrm{a}}$ & $47.2 \pm 17.3$ & $89.6 \pm 5.5$ & $<0.01$ \\
Quality of life $^{\mathrm{a}}$ & $24.1 \pm 23.0$ & $64.0 \pm 10.4$ & $<0.01$ \\
\hline
\end{tabular}

Values are presented as mean \pm standard deviation. $\mathrm{OA}$, osteoarthritis. ${ }^{a}$ Subscale for the Knee Osteoarthritis Outcome Survey. in Table 1. Both groups were similar in age, weight, height, waist circumference and body mass index $(\mathrm{p}>0.05)$.

Knee OA group showed greater values of lateral symmetry of COP $(\mathrm{p}<0.01)$ than healthy group. There were no significant differences in another gait and COP variables between groups $(\mathrm{p}>0.05)$ (Table 2).

With regarding to correlation's results, lateral symmetry of COP had moderate or strong correlation with the intensity of knee pain $(\rho=0.61, p<0.01)$, pain duration $(\rho=0.60$, p $<$ $0.01)$, symptom subscale $(\rho=-0.71, p<0.01)$, pain subscale $(\rho$ $=-0.58, p<0.01)$, activities of daily living subscale $(\rho=-0.70$, $p<0.01)$, function in sport and recreation subscale $(\rho=-0.63$, $p<0.01)$ and quality of life subscale of KOOS $(\rho=-0.56, p<$ 0.01). No significant or weak or moderate levels of correlation were noted between another gait or COP variables and clinical variables (Table 3).

\section{DISCUSSION}

Current study compared the between-limb asymmetry of gait and COP parameters between patients with knee OA and healthy controls and explored the association between knee $\mathrm{OA}$ related clinical variables and gait and COP parameters. Results from current study indicate that patients with radiographic knee OA demonstrate more asymmetry in lateral COPshift than healthy controls and significant correlation between clinical factors related knee OA and lateral symmetry of COP.

Previous researches have demonstrated between-limb kinematic and kinetic asymmetries in frontal planes of individuals with knee OA during walking [4,22,23]. Current study also demonstrated the asymmetry of lateral COP-shift in patients with knee OA, specifically COP of knee OA group was more shifted laterally toward symptomatic side $(\mathrm{p}<0.01)$ than

Table 2. Comparison of symmetry of gait parameters between groups

\begin{tabular}{|c|c|c|c|}
\hline Characteristics & $\begin{array}{l}\text { Knee OA } \\
\text { group }\end{array}$ & $\begin{array}{l}\text { Healthy control } \\
\text { group }\end{array}$ & p-value \\
\hline SI of angle of foot rotation (\%) & $68.2 \pm 66.1$ & $53.3 \pm 33.6$ & 0.71 \\
\hline SI of step length (\%) & $4.4 \pm 4.7$ & $5.7 \pm 4.7$ & 0.52 \\
\hline SI of stance phase (\%) & $5.8 \pm 5.0$ & $6.1 \pm 5.7$ & 0.14 \\
\hline SI of swing phase (\%) & $3.0 \pm 2.3$ & $3.8 \pm 2.0$ & 0.13 \\
\hline SI of length of gait line ${ }^{a}(\%)$ & $3.2 \pm 2.2$ & $4.0 \pm 2.1$ & 0.07 \\
\hline $\begin{array}{l}\text { Anterior/posterior position }{ }^{a} \\
\text { (mm) }\end{array}$ & $139.7 \pm 8.8$ & $140.8 \pm 9.1$ & 0.74 \\
\hline Lateral symmetry ${ }^{\mathrm{a}}(\mathrm{mm})$ & $3.1 \pm 7.3$ & $-2.6 \pm 2.2$ & $<0.01 *$ \\
\hline
\end{tabular}

Values are presented as mean \pm standard deviation. OA, osteoarthritis; $\mathrm{SI}$, symmetry index. ${ }^{\mathrm{a}}$ The variables of center of pressure. ${ }^{*} \mathrm{p}<0.05$. 
Table 3. Spearman's correlation coefficients between clinical measures and gait parameters

\begin{tabular}{|c|c|c|c|c|c|c|c|}
\hline \multirow{2}{*}{ Variable } & \multirow{2}{*}{ Intensity of pain } & \multirow{2}{*}{ Pain duration } & \multicolumn{5}{|c|}{ KOOS subscale } \\
\hline & & & Symptom & Pain & $A D L$ & Sport & QOL \\
\hline SI of angle of foot rotation & 0.12 (0.53) & $-0.03(0.86)$ & $-0.15(0.41)$ & $-0.22(0.24)$ & $-0.18(0.34)$ & $-0.22(0.24)$ & $-0.25(0.18)$ \\
\hline SI of step length & $0.11(0.56)$ & $0.09(0.63)$ & $-0.23(0.22)$ & $-0.16(0.39)$ & $-0.12(0.52)$ & $0.05(0.78)$ & $0.12(0.51)$ \\
\hline SI of stance phase & $0.22(0.23)$ & $0.38(0.04)$ & $-0.43 *(0.02)$ & $-0.31(0.09)$ & $-0.34(0.66)$ & $-0.30(0.10)$ & $-0.20(0.28)$ \\
\hline SI of swing phase & $0.13(0.48)$ & $0.34(0.06)$ & $-0.34(0.06)$ & $-0.15(0.40)$ & $-0.20(0.28)$ & $-0.25(0.18)$ & $-0.24(0.20)$ \\
\hline SI of length of gait line $e^{a}$ & $0.36(0.05)$ & $0.29(0.11)$ & $-0.20(0.27)$ & $-0.24(0.20)$ & $-0.28(0.14)$ & $-0.28(0.13)$ & $-0.38 *(0.04)$ \\
\hline Anterior/posterior position ${ }^{a}$ & $0.10(0.59)$ & $0.16(0.40)$ & $-0.18(0.32)$ & $-0.27(0.14)$ & $-0.17(0.35)$ & $-0.21(0.27)$ & $0.26(0.16)$ \\
\hline Lateral symmetry $^{\mathrm{a}}$ & $0.61 *(<0.01)$ & $0.60 *(<0.01)$ & $-0.71 *(<0.01)$ & $-0.58 *(<0.01)$ & $-0.70 *(<0.01)$ & $-0.63 *(<0.01)$ & $-0.56 *(<0.01)$ \\
\hline
\end{tabular}

Values are presented as $\rho$ ( $p$ value). SI, symmetry index; KOOS, Knee Osteoarthritis Outcome Survey; ADL, activities of daily living; QOL, quality of life. ${ }^{a}$ The variables of center of pressure. ${ }^{*} p<0.05$.

healthy control group. This result can be explained with compensatory ipsilateral trunk leaning toward symptomatic side of knee OA during walking. Patients with severe knee OA exhibited more ipsilateral trunk leaning toward more symptomatic side than healthy control and patients with moderate knee OA [22]. Excessive trunk lean during walking may be a natural compensatory mechanism to knee OA, and patients with knee OA adopt compensatory trunk leaning to receive the mechanical benefit during walking [3,24]. Even though current study did not measure the trunk motion during gait, patients with knee OA also may adopt trunk leaning strategy based on the results of lateral COP-shift towards the affected side. Because current study demonstrated asymmetrical lateral COP-shift towards to affected side in knee OA patients, it is feasible that this finding can be useful for physiotherapist when assessing the quality of gait and applying feedback training using pressure mat or pressure insole in order to reduce the lateral COPshift during gait.

Interestingly, current study found significant moderate or strong association between asymmetrical lateral COP-shift towards to symptomatic side during walking and knee OA related clinical variables. More lateral COP-shift occurred, more intensity and duration of knee pain got worse in correlation analysis in current study. In addition, more lateral COP-shift increase, more KOOS scores of pain, symptom, function in daily living/ sports and quality of life subscales also decreased, meaning getting worse of functional scores in results of KOOS. In previous results, amount of trunk leaning during walking have just weak correlation (Pearson's $r=0.25$ ) with WOMAC pain score in patients with unilateral and/or bilateral knee OA [22]. All subjects in current study had unilateral knee OA with no pain, symptom and radiological features of sound side, resulting higher correlation when comparing the correlation of the previous study
[22]. These findings mean that patients with knee OA tend to walk asymmetrically toward lateral direction as to level of pain severity and functional score of knee OA getting worse. Based on these significant relationships, clinicians tend to consider lateral symmetry of COP as a relevant clinical marker of pain severity and mobility deficits in knee OA. However, although current results provide a potential relationship between asymmetrical COP laterality and knee OA related pain/function, given that current study was cross-sectional design, we could not determine that any patients with knee OA increase the amount of lateral COP-shift as their pain intensity increase.

Although our subjects had unilateral knee OA, symmetry of the angle of foot rotation showed no significant differences between knee OA group and healthy controls and no significant association with any clinical variables. Adopting a toe-out strategy during walking in patients with knee $\mathrm{OA}$ is effective for the reduction of load on the medial compartment of knee joint as well as knee adduction moment, inducing to relieve pain [25]. However, healthy older females without knee OA walk with external rotation at the foot to reduce knee adduction moment during late stance phase, as well as patients with knee OA $[25,26]$. Similar toe-out pattern of foot progression in both older females with and without knee OA may lead to no significant difference. We also measured between-limb symmetry of step length and anterior/posterior position of COP, but found no significant differences between groups and no significant correlations with knee OA related pain and functional variables. Since there are higher associations between frontal plane motion of hip and ankle in knee OA patients rather than sagittal plane $[22,23,27]$, step length and anterior/ posterior position of COP intersection point between limbs, which were influenced by sagittal kinetic and kinematic of the hip and ankle joint, might not differ between OA group and 
healthy group, and there was no association with knee pain intensity and functional scores.

A limitation of current study was the relatively small sample size in current study when comparing the previous gait-related study [28,29]. A second limitation was that all subjects had only unilateral knee $\mathrm{OA}$ due to the purpose of the current study, investigating the symmetry or asymmetry. Thus, these findings cannot be generalized to patients with bilateral knee OA. Thirdly, current the study had a cross-sectional design, meaning we were unable to investigate the cause and effect relationship between lateral symmetry of COP and knee OArelated clinical variables. Future longitudinal and prospective cohort studies should be undertaken to further investigate this relationship in patients with knee OA. Lastly, since asymmetry of angle of foot pronation and hallux valgus can influence the asymmetry of foot pressure pattern as well as knee joint musculoskeletal elements [30,31], further study would be needed to specify the risk factors related to knee OA, including asymmetric foot posture.

\section{CONCLUSIONS}

Current results have clinical importance given that patients with radiographic unilateral knee $\mathrm{OA}$ and pain showed more asymmetry of lateral COP-shift during walking compared with matched healthy controls. In addition, decreased lateral symmetry of COP has moderate or strong correlation with increased knee pain, longer pain duration, and worse KOOS scores. Due to the cross-sectional nature of the current study, it is not difficult to figure out why laterality of COP between limb occur, however, it is possible that asymmetry of lateral COP-shift between limbs may be one of the characteristics of unilateral knee $\mathrm{OA}$ as the compensatory strategy response to knee OA.

\section{ACKNOWLEDGEMENTS}

This work has supported by the National Research Foundation of Korea (NRF) grant funded by the Korea government (MSIT) (No. 2020R1A2C2102729).

\section{CONFLICTS OF INTEREST}

No potential conflict of interest relevant to this article was reported.

\section{AUTHOR CONTRIBUTIONS}

Conceptualization: KP. Formal analysis: KP. Funding acquisition: KP. Investigation: KP. Methodology: KP, SK. Resources: KP. Supervision: KP, SK. Validation: KP. Visualization: KP, SK. Writing - original draft: SK. Writing - review \& editing: KP, SK.

\section{ORCID}

Si-hyun Kim, https://orcid.org/0000-0003-1870-2767

\section{REFERENCES}

1. Andriacchi TP, Lang PL, Alexander EJ, Hurwitz DE. Methods for evaluating the progression of osteoarthritis. J Rehabil Res Dev 2000;37(2):163-70.

2. Srikanth VK, Fryer JL, Zhai G, Winzenberg TM, Hosmer D, Jones G. A meta-analysis of sex differences prevalence, incidence and severity of osteoarthritis. Osteoarthritis Cartilage 2005;13(9):769-81.

3. Mündermann A, Dyrby CO, Andriacchi TP. Secondary gait changes in patients with medial compartment knee osteoarthritis: increased load at the ankle, knee, and hip during walking. Arthritis Rheum 2005;52(9):2835-44.

4. Briem K, Snyder-Mackler L. Proximal gait adaptations in medial knee OA. J Orthop Res 2009;27(1):78-83.

5. Pincus T, Castrejon I, Yazici Y, Gibson KA, Bergman MJ, Block JA. Osteoarthritis is as severe as rheumatoid arthritis: evidence over 40 years according to the same measure in each disease. Clin Exp Rheumatol 2019;37 Suppl 120(5):7-17.

6. Mills K, Hettinga BA, Pohl MB, Ferber R. Between-limb kinematic asymmetry during gait in unilateral and bilateral mild to moderate knee osteoarthritis. Arch Phys Med Rehabil 2013;94(11):2241-7.

7. Sant'Anna A, Salarian A, Wickström N. A new measure of movement symmetry in early Parkinson's disease patients using symbolic processing of inertial sensor data. IEEE Trans Biomed Eng 2011;58(7):2127-35.

8. Sadeghi H, Allard P, Prince F, Labelle H. Symmetry and limb dominance in able-bodied gait: a review. Gait Posture 2000;12(1):34-45.

9. Creaby MW, Bennell KL, Hunt MA. Gait differs between unilat- 
eral and bilateral knee osteoarthritis. Arch Phys Med Rehabil 2012;93(5):822-7.

10. Altman R, Asch E, Bloch D, Bole G, Borenstein D, Brandt K, et al. Development of criteria for the classification and reporting of osteoarthritis. Classification of osteoarthritis of the knee. Diagnostic and Therapeutic Criteria Committee of the American Rheumatism Association. Arthritis Rheum 1986;29(8):103949.

11. Ferrigno C, Wimmer MA, Trombley RM, Lundberg HJ, Shakoor $\mathrm{N}$, Thorp LE. A reduction in the knee adduction moment with medial thrust gait is associated with a medial shift in center of plantar pressure. Med Eng Phys 2016;38(7):615-21.

12. Lidtke RH, Muehleman C, Kwasny M, Block JA. Foot center of pressure and medial knee osteoarthritis. J Am Podiatr Med Assoc 2010;100(3):178-84.

13. Viton JM, Timsit M, Mesure S, Massion J, Franceschi JP, Delarque A. Asymmetry of gait initiation in patients with unilateral knee arthritis. Arch Phys Med Rehabil 2000;81 (2):194-200.

14. Resende RA, Kirkwood RN, Deluzio KJ, Morton AM, Fonseca ST. Mild leg length discrepancy affects lower limbs, pelvis and trunk biomechanics of individuals with knee osteoarthritis during gait. Clin Biomech (Bristol, Avon) 2016;38:1-7.

15. Henriksen M, Christensen R, Klokker L, Bartholdy C, Bandak E, Ellegaard K, et al. Evaluation of the benefit of corticosteroid injection before exercise therapy in patients with osteoarthritis of the knee: a randomized clinical trial. JAMA Intern Med 2015;175(6):923-30.

16. Roos EM, Toksvig-Larsen S. Knee injury and Osteoarthritis Outcome Score (KOOS) - validation and comparison to the WOMAC in total knee replacement. Health Qual Life Outcomes 2003;1:17.

17. Faude O, Donath L, Roth R, Fricker L, Zahner L. Reliability of gait parameters during treadmill walking in communitydwelling healthy seniors. Gait Posture 2012;36(3):444-8.

18. Marot V, Murgier J, Carrozzo A, Reina N, Monaco E, Chiron P, et al. Determination of normal KOOS and WOMAC values in a healthy population. Knee Surg Sports Traumatol Arthrosc 2019;27(2):541-8.

19. Lee DY, Seo SG, Kim EJ, Lee DJ, Bae KJ, Lee KM, et al. Inter-segmental motions of the foot: differences between younger and older healthy adult females. J Foot Ankle Res 2017;10:29.

20. Wearing SC, Reed LF, Urry SR. Agreement between temporal and spatial gait parameters from an instrumented walkway and treadmill system at matched walking speed. Gait Posture
2013;38(3):380-4.

21. Patterson KK, Gage WH, Brooks D, Black SE, McIlroy WE. Evaluation of gait symmetry after stroke: a comparison of current methods and recommendations for standardization. Gait Posture 2010;31(2):241-6.

22. Hunt MA, Wrigley TV, Hinman RS, Bennell KL. Individuals with severe knee osteoarthritis (OA) exhibit altered proximal walking mechanics compared with individuals with less severe OA and those without knee pain. Arthritis Care Res (Hoboken) 2010;62(10):1426-32.

23. Hunt MA, Birmingham TB, Giffin JR, Jenkyn TR. Associations among knee adduction moment, frontal plane ground reaction force, and lever arm during walking in patients with knee osteoarthritis. J Biomech 2006;39(12):2213-20.

24. Huang SC, Wei IP, Chien HL, Wang TM, Liu YH, Chen HL, et al. Effects of severity of degeneration on gait patterns in patients with medial knee osteoarthritis. Med Eng Phys 2008;30(8): 997-1003.

25. Guo M, Axe MJ, Manal K. The influence of foot progression angle on the knee adduction moment during walking and stair climbing in pain free individuals with knee osteoarthritis. Gait Posture 2007;26(3):436-41.

26. Teichtahl AJ, Morris ME, Wluka AE, Baker R, Wolfe R, Davis SR, et al. Foot rotation--a potential target to modify the knee adduction moment. J Sci Med Sport 2006;9(1-2):67-71.

27. Levinger P, Menz HB, Morrow AD, Feller JA, Bartlett JR, Bergman NR. Foot kinematics in people with medial compartment knee osteoarthritis. Rheumatology (Oxford) 2012;51(12): 2191-8.

28. Mills K, Hunt MA, Ferber R. Biomechanical deviations during level walking associated with knee osteoarthritis: a systematic review and meta-analysis. Arthritis Care Res (Hoboken) 2013; 65(10):1643-65.

29. Elbaz A, Mor A, Segal G, Debi R, Shazar N, Herman A. Novel classification of knee osteoarthritis severity based on spatiotemporal gait analysis. Osteoarthritis Cartilage 2014;22(3): 457-63.

30. Wen J, Ding Q, Yu Z, Sun W, Wang Q, Wei K. Adaptive changes of foot pressure in hallux valgus patients. Gait Posture 2012; 36(3):344-9.

31. Kim S, You K, Jung D. Between-side comparisons of iliotibial band flexibility and the tibial torsion angle in subjects with an asymmetric hallux valgus angle. J Musculoskelet Sci Technol 2018;2(1):11-5. 